Pacific Journal of Mathematics

THE PROPER FORCING AXIOM AND STATIONARY SET 


\title{
THE PROPER FORCING AXIOM AND STATIONARY SET REFLECTION
}

\author{
ROBERT E. BEAUdOIN
}

\begin{abstract}
Our main result is that the proper forcing axiom (PFA) is equiconsistent with "PFA + there is a nonreflecting stationary subset of $\omega_{2}$." More generally we show for any cardinals $n<m \leq \aleph_{2}$ that if $\mathrm{PFA}^{+}(n)$ is consistent with ZFC then so is " $\mathrm{PFA}^{+}(n)+$ there are $m$ mutually nonreflecting stationary subsets of $\omega_{2}$." As corollaries we can show that if $n<m \leq \aleph_{1}$ then $\operatorname{PFA}^{+}(n)$ (if consistent) does not imply $\operatorname{PFA}^{+}(m)$, and that PFA (if consistent) does not imply Martin's's maximum.
\end{abstract}

1. Introduction. Recently much attention has been given to various strengthenings of Martin's axiom for $\aleph_{1}$. Following [FMS] let us denote by $\operatorname{MA}(\Gamma)$, where $\Gamma$ is a class of partial orders, the statement:

If $P \in \Gamma$ and $\Delta$ is a family of at most $\aleph_{1}$ dense subsets of $P$, then there is a $\Delta$-generic filter on $P$.

Thus letting $\Gamma$ be the class of all partial orders having the c.c.c., $\operatorname{MA}(\Gamma)$ becomes Martin's axiom (for $\aleph_{1}$ dense sets). Taking $\Gamma$ to be the class of proper partial orders, $\operatorname{MA}(\Gamma)$ becomes the proper forcing axiom (PFA). Taking $\Gamma$ to be the class of all orders $P$ so that forcing the $P$ preserves the stationarity of subsets of $\omega_{1}, \operatorname{MA}(\Gamma)$ becomes Martin's maximum (MM), discussed in [FMS].

One may fortify these axioms by demanding that the filter obtained not only be generic, but also respect the stationarity of a collection of subsets (in the generic extension) of $\omega_{1}$. That is, one may consider the axioms

$\mathrm{MA}^{+}(\Gamma, \kappa):$ If $P \in \Gamma, \Delta$ is a family of at most $\aleph_{1}$ dense subsets of $P$, and $\left\{\mathbf{S}_{\alpha}: \alpha<\kappa\right\}$ is a family of terms, each forced by every condition in $P$ to denote a stationary subset of $\omega_{1}$, then there is a $\Delta$-generic filter $G$ on $P$ so that for every $\alpha<\kappa, \mathbf{S}_{\alpha}(G)$ is stationary in $\omega_{1}$.

(Here $\mathbf{S}_{\alpha}(G)=\left\{\beta<\omega_{1}: \exists p \in G p \Vdash\right.$ “ $\beta \in \mathbf{S}_{\alpha}$ " $\}$, the interpretation of the term $\mathbf{S}_{\alpha}$ by the filter $G$.) If $\Gamma$ is the class of proper 
partial orders $\mathrm{MA}^{+}(\Gamma, \kappa)$ is commonly called $\mathrm{PFA}^{+}(\kappa)$. The usual consistency proof of PFA in fact establishes consistency of $\mathrm{PFA}^{+}\left(\aleph_{1}\right)$ with ZFC (relative to the consistency of ZFC + "There is a supercompact cardinal"); it is not hard to see that $\operatorname{PFA}^{+}\left(\aleph_{2}\right)$ is inconsistent with ZFC. Baumgartner conjectured in [Ba] that $\mathrm{PFA}^{+}(1)$ is actually stronger than PFA; on the other hand, he also proved that if $\Gamma$ is the class of partial orders having the c.c.c., then $\operatorname{MA}(\Gamma)$ (i.e., ordinary $\left.\mathrm{MA}_{\aleph_{1}}\right)$ and $\mathrm{MA}^{+}(\Gamma, n)$ are equivalent for all finite $n$. Shelah [Sh, Remark 6A3] has proved that $\mathrm{MM}^{+}(n)$, if consistent, does not imply $\operatorname{PFA}^{+}(m) \quad\left(n<m \leq \aleph_{2}\right.$, both cardinals $)$, more than establishing Baumgartner's conjecture. Simpler proofs were given by Veličković [V]. Our arguments, announced at the Southeastern Logic Symposium (Gainesville, February 1985), only show that $\operatorname{PFA}^{+}(n)$, if consistent, does not imply $\operatorname{PFA}^{+}(m)$, but are considerably simpler still. Our methods also establish that PFA does not (if consistent) imply MM (as, of course, do Shelah's and Veličković's).

The wedge we will drive between PFA and $\mathrm{PFA}^{+}(1)$ is a nonreflecting stationary set. A stationary set $S$ on a cardinal $\kappa$ reflects if there is a limit $\alpha<\kappa$ with $\mathrm{cf} \alpha>\omega$ such that $S \cap \alpha$ is stationary in $\alpha$; we shall say $S$ reflects at $\alpha$. Let us call a family $F$ of stationary sets on $\kappa$ mutually reflecting if for some limit $\alpha<\kappa$ every $S \in F$ reflects at $\alpha$; otherwise we call $F$ mutually nonreflecting. For brevity's sake, we adopt Shelah's notation for sets of ordinals of specified cofinality: $S_{k}^{j}=\left\{\alpha<\omega_{j}:\right.$ cf $\left.\alpha=\omega_{k}\right\}$. Our main result (Theorem 2.6) is that for any $n<m, \operatorname{PFA}^{+}(n)$ (if consistent with $\mathrm{ZFC}$ ) is consistent with the existence of a family of $m$ mutually nonreflecting stationary subsets of $S_{0}^{2}$. But (trivially generalizing a theorem from [Ba] for one stationary set), letting $\Gamma$ be the class of all countably closed partial orders, $\mathrm{MA}^{+}(\Gamma, m)$ implies that every family of $m$ stationary subsets of $\omega_{2}$ is mutually reflecting. So $\operatorname{PFA}^{+}(n)$ is weaker than $\mathrm{PFA}^{+}(m)$. In independent (unpublished) work along the same lines, Magidor constructed, by forcing over a model with a supercompact cardinal, a model of set theory in which PFA holds and there is a nonreflecting stationary subset of $\omega_{2}$ (so $\operatorname{PFA}^{+}(1)$ fails). His argument is essentially the same as ours.

As another application of Theorem 2.6, we can derive (modulo consistency of PFA) the following theorem from our doctoral dissertation [Be] (unpublished): There is a model of ZFC in which there are no $\omega_{2}$-Aronszajn threes but there is a nonreflecting stationary subset of $S_{0}^{2}$. This follows from Theorem 2.6 (with $n=0$ and $m=1$ ) and 
Baumgartner's result (see [To] for a proof) that PFA implies there are no $\omega_{2}$-Aronszajn trees. In $[\mathrm{Be}]$ we obtained such a model by forcing over a model with a weakly compact cardinal; the proof of Theorem 2.6 is an elaboration of this earlier construction.

Finally, let us mention an open question. In [Sh], Shelah has shown that $\mathrm{MM}$ implies $\mathrm{MA}^{+}(\Gamma, 1)$, for $\Gamma$ the class of countably closed partial orders. We do not know if $\mathrm{MM}$ implies $\mathrm{MA}^{+}(\Gamma, m)$ for $m$ between 2 and $\aleph_{1}$ (and the same $\Gamma$ ). Question: For which $m \leq$ $\aleph_{2}$ does MM imply that every family of $m$ stationary subsets of $S_{0}^{2}$ is mutually reflecting? We cannot rule out the possibility that MM is consistent with the existence of a pair of mutually nonreflecting stationary subsets of $S_{0}^{2}$.

2. Adjoining mutually nonreflecting stationary sets. Our main goal is to show, for any cardinals $n$ and $m$ with $n<m \leq \aleph_{2}$, that $\operatorname{PFA}^{+}(n)$ is equiconsistent with $\operatorname{PFA}^{+}(n)+$ "There is a mutually nonreflecting family of $m$ stationary subset of $\omega_{2}$ ". Starting from a model of $\operatorname{PFA}^{+}(n)$ we will force to add such a family. Let $P$ be the set of all functions $p$ from $m$ into $\mathscr{P}\left(\omega_{2}\right)$, such that there is a $\beta<\omega_{2}$ so that for every $i<m p(i) \subseteq \beta \cap S_{0}^{2}$, for every $\alpha \in S_{1}^{2}, p(i) \cap \alpha$ is nonstationary in $\alpha$, and for $j \neq i \quad p(i) \cap p(j)=\varnothing$. For each $p \in P$ let $\operatorname{lh}(p)=\inf \{\beta: \forall i<m p(i) \subseteq \beta\}$, and call this ordinal the length of $p$. Endow $P$ with a partial ordering: $p \leq q$ iff for every $i<m$, $p(i) \cap \ln (q)=q(i)$.

LEMMA 2.1. $P$ is countably complete and $\omega_{2}$-Baire, and any $V$ generic filter on $P$ is equiconstructible over $V$ with an enumeration $E$ of a family of $m$ pairwise disjoint, mutually nonreflecting stationary subsets of $S_{0}^{2}$, whose union is costationary in $S_{0}^{2}$.

Proof. If $\left\langle p_{k}: k\langle\omega\rangle\right.$ is a decreasing sequence in $P$ then defining $p(i)=\bigcup\left\{p_{k}(i): k<\omega\right\}$ for each $i<m$ clearly makes $p$ the infimum of $\left\{p_{n}: n<\omega\right\}$ in $P$. So $P$ is countably complete.

Next, suppose that $\left\langle D_{\sigma}: \sigma<\omega_{1}\right\rangle$ is a sequence of dense open subsets of $P$, and that $p \in P$ is arbitrary. We shall find $q \leq p$ such that $q \in \bigcap\left\{D_{\sigma}: \sigma<\omega_{1}\right\}$. Construct $\left\langle\left(p_{\sigma}, \alpha_{\sigma}\right): \sigma<\omega_{1}\right\rangle$ by induction on $\sigma$ such that:

(1) $p_{0}=p$ and $\alpha_{0}=\operatorname{lh}(p)+1$.

(2) $p_{\sigma+1} \leq p_{\sigma}, p_{\sigma+1} \in D_{\sigma}, \operatorname{lh}\left(p_{\sigma+1}\right)>\alpha_{\sigma}$, and $\alpha_{\sigma+1}=\operatorname{lh}\left(p_{\sigma+1}\right)$ +1 . 
(3) If $\sigma$ is limit then $\alpha_{\sigma}=\sup \left\{\alpha_{\tau}: \tau<\sigma\right\}$, for some $i<$ $m p_{\sigma}(i)=\bigcup\left\{p_{\tau}(i): \tau<\sigma\right\} \cup\left\{\alpha_{\sigma}+\omega\right\}$, and for the remaining $j \neq i p_{\sigma}(j)=\bigcup\left\{p_{\tau}(j): \tau<\sigma\right\}$.

So for limit $\sigma p_{\sigma}$ is a condition of length $\alpha_{\sigma}+\omega$ extending each $p_{\tau}$, such that $\alpha_{\sigma} \notin \bigcup\left\{p_{\sigma}(i): i<m\right\}$. Note for any $\sigma$ that $\alpha_{\sigma+1}$, being a successor ordinal, is not in $\bigcup\left\{p_{\sigma+2}(i): i<m\right\}$, whence for any $\sigma$ and $\tau, \alpha_{\sigma} \notin \bigcup\left\{p_{\tau}: i<m\right\}$. For each $i<m$ let $q(i)=\bigcup\left\{p_{\sigma}(i): i<m\right\}$, and let $\alpha=\sup \left\{\alpha_{\sigma}: \sigma<\omega_{1}\right\}$. Then $\left\{\alpha_{\sigma}: \sigma<\omega_{1}\right\}$ is club in $\alpha$ and disjoint from $\bigcup\{q(i): i<m\}$, and it follows easily that $q$ is a condition in $P$. Clearly $q \leq p_{\sigma}$ for each $\sigma$, so $q \leq p$ belongs to each $D_{\sigma}$, which shows that $P$ is $\omega_{2}$-Baire.

Now suppose $F$ is a $V$-generic filter on $P$, and define a function $E$ with domain $m$ by setting $E(i)=\bigcup\{p(i): p \in F\}$. As $F=$ $\{p \in P: \forall i<m p(i)=E(i) \cap \operatorname{lh}(p)\}, F$ and $E$ are equiconstructible. Clearly $E$ enumerates a family of $m$ pairwise disjoint sets, and for each $\alpha<\omega_{2}$ and $i<m E(i) \cap \alpha$ is nonstationary in $\alpha$. We claim that each $E(i)$ is stationary in $\omega_{2}$. Supposing $p \in P$ and $p \Vdash$ "C is club in $\omega_{2}$, , it is enough to find $q \leq p$ and $\alpha \in q(i)$ such that $q \Vdash$ " $\alpha \in \mathbf{C}$."

Fix $\lambda$ regular and so large that $P \in H(\lambda)$, and let $<^{*}$ well-order $H(\lambda)$. Choose a countable elementary substructure $N$ of $\left(H(\lambda), \varepsilon,<^{*}\right)$ such that $p, P, \mathbf{C} \in N$. (The name $\mathbf{C}$ may be identified with $\{(r, \beta): r \Vdash " \beta \in \mathbf{C} "\}$.) Let $\alpha=\sup \left(N \cap \omega_{2}\right)$. Choose a descending sequence $\left\langle p_{k}: k<\omega\right\rangle$ of conditions in $P \cap N$ so that $p_{0}=p$ and for every $D \in N$, if $D \subseteq P$ is dense below $p$ then $p_{k} \in D$ for some $k$. Let $p^{\prime}=\inf \left\{p_{k}: k<\omega\right\}$. Simple dense-set arguments show $\operatorname{lh}\left(p^{\prime}\right)=\alpha$ and $p^{\prime} \Vdash$ “ $\alpha$ is a limit point of $\mathbf{C}$." Thus $p^{\prime} \Vdash " \alpha \in \mathbf{C}$." Define $q$ by setting $q(i)=p^{\prime}(i) \cup\{\alpha\}$, and setting $q(j)=p(j)$ for $j \neq i$. Clearly $q$ is a condition such that $q \leq p, \alpha \in q(i)$, and $q \Vdash$ " $\alpha \in \mathbf{C}$," as desired.

Finally we must show that $S_{0}^{2}-\bigcup\{E(i): i<m\}$ is stationary. It suffices, given $p \in P$ such that $p \Vdash$ "C is club in $\omega_{2}$," to find $q \leq p$ and $\alpha \in S_{0}^{2}$ such that $q \Vdash$ " $\alpha \in \mathbf{C}$ " and $\alpha<\operatorname{lh}(q)$, yet $\alpha \notin \bigcup\{q(i): i<m\}$. As above we can find $p^{\prime}$ and $\alpha$ so that $p^{\prime} \leq p$, $\ln \left(p^{\prime}\right)=\alpha \in S_{0}^{2}$, and $p^{\prime} \Vdash$ “ $\alpha \in \mathbf{C}$." Now choose some $i<m$, let $q(i)=p^{\prime}(i) \cup\{\alpha+\omega\}$, and for $j \neq i$ let $q(j)=p^{\prime}(j)$. Clearly $q \in P$ is as desired, and so the lemma is proved.

REMARK. If $m=1$ then forcing with $P$ adjoins a single nonreflecting stationary subset of $S_{0}^{2}$ which is also costationary in $S_{0}^{2}$. 
Now our main result is easy to state: If $V$ is a model of set theory plus $\mathrm{PFA}^{+}(n)$ then any generic extension of $V$ by forcing with $P$ is also a model of $\mathrm{PFA}^{+}(n)$. Hence $\mathrm{PFA}^{+}(n)$ and $\mathrm{PFA}^{+}(n)+$ "there are $m$ mutually nonreflecting stationary subsets of $\omega_{2}$ " are equiconsistent (over ZFC). For use in the proof, we establish the following notation and terminology. $E$ will always be a family constructed as in Lemma 2.1 from a $V$-generic filter on $P$, and $\mathbf{E}$ will be the canonical $P$-term for such a family. Thus any generic extension of $V$ by $P$ has the form $V[E]$. ( $E$ and the generic filter are equiconstructible.) Note that forcing with $P$ preserves $\aleph_{1}$ and $\aleph_{2}$, so $n$ and $m$ are not collapsed. Note also that if $p \in P, \alpha \in S_{0}^{2}$, and for some $i<m$ either $p \Vdash$ " $\alpha \in \mathbf{E}(i)$ " or $p \Vdash$ " $\alpha \notin \mathbf{E}(i)$ " then $\alpha<\operatorname{lh}(p)$.

We shall call $\left(Q, \Delta,\left\langle\mathbf{S}_{i}: i<n\right\rangle\right)$ an obnoxious triple if $Q$ is a proper partial order, $\Delta$ a family of at most $\aleph_{1}$ dense subsets of $Q$, and each $\mathbf{S}_{i}$ a $Q$-term for a stationary subset of $\omega_{1}$, such that there is no $\Delta$-generic filter $G$ on $Q$ for which $\mathbf{S}_{i}(G)$ is stationary for every $i<n$. Call $Q$ obnoxious if there are a $\Delta$ and $\left\langle\mathbf{S}_{i}: i<n\right\rangle$ so that $\left(Q, \Delta,\left\langle\mathbf{S}_{i}: i<n\right\rangle\right)$ is an obnoxious triple. So $\operatorname{PFA}^{+}(n)$ states that there is no obnoxious $Q$.

LEMMA 2.2. If there is an obnoxious $Q$ then there is an obnoxious triple $\left(Q^{\prime}, \Delta,\left\langle\mathbf{S}_{i}: i<n\right\rangle\right)$ so that $Q^{\prime}$ collapses $\aleph_{2}$ to cardinality $\aleph_{1}$ and each $D \in \Delta$ is dense and open in $Q^{\prime}$.

Proof. Suppose $\left(Q,\left\{F_{\sigma}: \sigma<\omega_{1}\right\},\left\langle\mathbf{S}_{i}: i<n\right\rangle\right)$ is an obnoxious triple. If $Q$ does not collapse $\aleph_{2}$ let $C$ be the Levy partial order for adding a function from $\omega_{1}$ onto $\omega_{2}$ with countable conditions, defined in the extension by $Q$, and if $Q$ does collapse $\aleph_{2}$ let $C$ be the trivial order. Since $C$ is countably closed, $Q * C$ is proper. Let $D_{\sigma}=\left\{(q, c) \in Q * C: \exists q^{\prime} \in F_{\sigma} q \leq q^{\prime}\right\}$. Clearly $D_{\sigma}$ is dense open and if $G$ were a $\left\{D_{\sigma}: \sigma<\omega_{1}\right\}$-generic filter on $Q * C$ then $\{q: \exists c(q, c) \in G\}$ would be $\left\{F_{\sigma}: \sigma<\omega_{1}\right\}$-generic on $Q$. So $\left(Q * C,\left\{D_{\sigma}: \sigma<\omega_{1}\right\},\left\langle\mathbf{S}_{i}: i<n\right\rangle\right)$ is an obnoxious triple.

The following is Lemma 8.2 of [Ba].

LEMMA 2.3. If $\alpha$ is an ordinal of uncountable cofinality and $S \subseteq$ $\{\beta<\alpha: \operatorname{cf} \beta=\omega\}$, then $S$ is stationary in $\alpha$ iff $\left\{x \in[\alpha]^{\leq \kappa_{0}}\right.$ : $\sup (x) \in S\}$ is stationary in $[\alpha]^{\leq \aleph_{0}}$. 
Using Lemma 2.3 it is easy to show that proper partial orders preserve stationarity of sets of ordinals of countable cofinality. (This is well known, but as we have no reference we include the simple proof.)

LEMMA 2.4. If $Q$ is a proper partial order, $\alpha$ is regular and uncountable, and $S \subseteq\{\beta<\alpha: \operatorname{cf} \beta=\omega\}$ is stationary, then $\Vdash_{Q}$ " $S$ is stationary in $\alpha$. ." (Note that $Q$ may collapse $\alpha$, though in the extension by $Q \alpha$ must remain uncountable.)

Proof. By Lemma $2.3\left\{x \in[\alpha]^{\leq \aleph_{0}}: \sup (x) \in S\right\}$ is stationary, and as $Q$ is proper this set remains stationary in the extension by $Q$. But then applying Lemma 2.3 again in $V^{Q}$ shows that $S$ remains stationary there.

Now suppose $E$ is a mutually nonreflecting family of stationary sets added to $V$ by forcing with $P, Q$ is a proper partial order in $V[E]$ that collapses $\aleph_{2}$, and $G$ is $V[E]$-generic on $Q$. In $V[E, G] \omega_{2}^{V}$ has a club subset of order type $\omega_{1}$; let $f$ be the increasing enumeration of such a club. After forcing with $Q$ each $E(i)$ remains stationary in $\omega_{2}$ by Lemma 2.4 , so $f^{-1 "} E(i)$ is stationary in $\omega_{1}$; let $R_{i}$ be the usual partial order for shooting a club through the complement of $f^{-1 "} E(i)$. That is, $R_{i}$ is the set of all continuous, strictly increasing functions $r$ whose domains are countable successor ordinals and whose ranges are subsets of $\omega_{1}-f^{-1 \prime} E(i)$. Since $R_{i}$ adjoins a club subset $C$ of $\omega_{1}$ such that $f^{\prime \prime} C$ is disjoint from $E(i)$ and $f^{\prime \prime} C$ is club in $\omega_{2}, R_{i}$ is not proper. Central to the arguments of this section is the following lemma.

LEMMA 2.5. Let $E, Q, f, i$ and $R_{i}$ be as above. Let $\mathbf{f} \in V$ be any $P * Q$-term for $f$, such that every condition forces $\mathbf{f}$ to denote the increasing enumeration of a club in $\omega_{2}$ of type $\omega_{1}$. Then:

(a) The iteration $P * Q * R_{i}$ is a proper partial order.

(b) For all sufficiently large regular $\lambda$ there is a $D$ club in $[H(\lambda)]^{\leq \aleph_{0}}$ such that if $N \in D,(p, q, r) \in P * Q * R_{i} \cap N, \alpha=N \cap \omega_{1}$, and $\zeta=\sup \left(N \cap \omega_{2}\right)$, then there is an extension $\left(p^{\prime}, q^{\prime}, r^{\prime}\right)$ of $(p, q, r)$

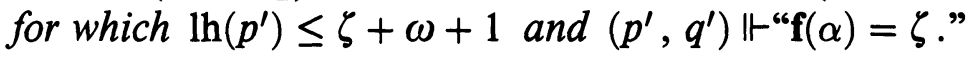

Proof. (a) Fix $\lambda$ regular and so large that $\mathbf{f}$ and the power set of $P * Q * R_{i}$ both belong to $H(\lambda)$. Let $N \prec H(\lambda)$ be countable with $P * Q * R_{i}, \mathrm{f} \in N$, and let $(p, q, r) \in P * Q * R \cap N$ be arbitrary; it 
suffices to find an $\left(N, P * Q * R_{i}\right)$-generic extension of $(p, q, r)$. Let $\alpha=N \cap \omega_{1}$ and $\zeta=\sup \left(N \cap \omega_{2}\right)$. Of course $\alpha \in \omega_{1}$ and $\zeta \in S_{0}^{2}$.

Begin by choosing $p^{\prime} \leq p$ an $(N, P)$-generic condition as follows. Let $\left\langle D_{k}: k<\omega\right\rangle$ enumerate the dense subset of $P$ contained in $N$, and define a descending sequence $\left\langle p_{k}: k<\omega\right\rangle$ of conditions in $P$ such that $p_{0}=p$ and for each $k, p_{k+1} \in D_{k} \cap N$. Let $p^{*}=$ $\inf \left\{p_{k}: k<\omega\right\}$. Then $\operatorname{lh}\left(p^{*}\right)=\zeta$. Let $p^{\prime}(i)=p^{*}(i) \cup\{\zeta+\omega\}$, and for $j \neq i$ let $p^{\prime}(j)=p^{*}(j)$. Then $p^{\prime} \in P$ is $(N, P)$-generic, $p^{\prime} \leq p$, $\ln \left(p^{\prime}\right)=\zeta+\omega+1$, and $p^{\prime} \Vdash$ " $\zeta \notin \mathbf{E}(i)$."

Since $\Vdash$ " $Q$ is proper", there is a term $q^{\prime}$ for a condition in $Q$ such that $p^{\prime} \Vdash$ " $q$ ' $\leq q$ and $q^{\prime}$ is $(N[\mathrm{E}], Q)$-generic." Then $\left(p^{\prime}, q^{\prime}\right)$ is an $(N, P * Q)$-generic extension of $(p, q)$. It follows that $\left(p^{\prime}, q^{\prime}\right) \Vdash$ " $N[\mathbf{E}, \mathbf{G}] \cap \omega_{1}=\alpha$ and $\sup \left(N[\mathbf{E}, \mathbf{G}] \cap \omega_{2}^{V}\right)=\zeta$." (Here of course $\mathbf{G}$ is a term denoting the $V[E]$-generic filter on $Q$.) Work for the moment in an extension $V[E, G]$, where $E$ is constructed from a $V$ generic filter on $P$ containing the condition $p^{\prime}, G$ is $V[E]$-generic on $Q$, and $q^{\prime} \in G$. Then $N[E, G]$ is a countable elementary substructure of $H(\lambda)^{V[E, G]}$ containing $R_{i}$ and $r$, with $N[E, G] \cap \omega_{1}=\alpha$ and $\sup \left(N[E, G] \cap \omega_{2}\right)=\zeta \notin E(i)$. As $\mathbf{f} \in N, f \in N[E, G]$. It follows by elementarity that $\sup f^{\prime \prime}(\alpha)=\zeta$, and by continuity $f(\alpha)=\zeta$.

Let $\left\langle F_{k}: k<\omega\right\rangle$ enumerate the dense subsets of $R_{i}$ belonging to $N[E, G]$. Using the elementarity of $N[E, G]$ we can choose a descending sequence $\left\langle r_{k}: k\langle\omega\rangle\right.$ of conditions in $R_{i}$, so that $r_{0}=r$ and $r_{k+1} \in F_{k} \cap N[E, G]$. Let $r^{*}=\bigcup\left\{r_{k}: k<\omega\right\} \cup\{(\alpha, \alpha)\}$. Clearly $r^{*}$ is increasing and continuous, and as $f(\alpha)=\zeta \notin E(i), r^{*}$ belongs to $R_{i}$. Clearly $r^{*}$ extends each $r_{k}$, and so $r^{*}$ is an $\left(N[E, G], R_{i}\right)$ generic extension of $r$.

Returning to $V$, we can find a term $r^{\prime}$ denoting $r^{*}$. Since $E$ and $G$ were arbitrary such that $q^{\prime} \in G$ and $E$ is the union of a generic filter containing $p^{\prime}$, we can choose $r^{\prime}$ so that $\left(p^{\prime}, q^{\prime}\right) \Vdash{ }^{\prime}$ ' $r^{\prime}$ is $\left(N[\mathbf{E}, \mathbf{G}], R_{i}\right)$-generic." It follows that $\left(p^{\prime}, q^{\prime}, r^{\prime}\right)$ is an $\left(N, P * Q * R_{i}\right)$-generic extension of $(p, q, r)$, which completes the proof that $P * Q * R_{i}$ is proper.

(b) Let $\lambda$ be as in the proof of (a) and let $D$ be the set of all countable elementary substructures of $H(\lambda)$ having $\mathrm{F}$ and $P * Q * R_{i}$ as elements. Given $N \in D$ and $(p, q, r) \in P * Q * R_{i} \cap N$, let $\alpha=N \cap \omega_{1}$ and $\zeta=\sup \left(N \cap \omega_{2}\right)$, and choose $\left(p^{\prime}, q^{\prime}, r^{\prime}\right)$ extending $(p, q, r)$ as in part (a). Clearly $\operatorname{lh}\left(p^{\prime}\right)=\zeta+\omega+1$. In the proof of part (a) we saw that for any generic extension $V[E, G]$ such that $p^{\prime}$ belongs to the generic filter on $P$ and $q^{\prime}$ belongs to $G, V[E, G] \vDash$ " $f(\alpha)=\zeta . "$ Hence $\left(p^{\prime}, q^{\prime}\right) \vDash$ "f $(\alpha)=\zeta . "$ 
Now we can prove the main result.

THEOREM 2.6. If $\mathrm{PFA}^{+}(n)$ holds in $V$ then $\vDash p$ "PFA ${ }^{+}(n)$." Thus if $\mathrm{PFA}^{+}(n)$ is consistent with ZFC then so is "PFA' $(n)+$ there is a family of $m$ mutually nonreflecting stationary subsets of $\omega_{2} . "$

Proof. Supposing the contrary, there must be a $P$-term $Q$ and a condition $p_{0}$, so that $p_{0} \Vdash$ " $Q$ is an obnoxious partial order." Since $\left\{p \in P: p \leq p_{0}\right\}$ is isomorphic to $P$, we may assume $p_{0}$ is the trivial condition. By Lemma 2.2 we may assume that forcing with $Q$ collapses $\aleph_{2}$ to cardinality $\aleph_{1}$, and find $P$-terms $\left\langle\mathbf{D}_{\sigma}: \sigma<\omega_{1}\right\rangle$ and $P * Q$-terms $\left\langle\mathbf{S}_{i}: i<n\right\rangle$ such that

$$
\Vdash^{\prime} \text { " }\left(Q,\left\langle\mathbf{D}_{\sigma}: \sigma<\omega_{1}\right\rangle,\left\langle\mathbf{S}_{i}: i<n\right\rangle\right. \text { is an obnoxious triple, " }
$$

and $\Vdash$ " $\mathbf{D}_{\sigma}$ is dense open" for each $\sigma$. As above, let $\mathbf{E}$ be a $P$-term for the mutually nonreflecting stationary family added by $P$, and let f be a $P * Q$-term, forced by every condition to denote the increasing enumeration of a club in $\omega_{2}^{V}$ of order type $\omega_{1}$. We may define the partial orders $R_{i}$ as in the discussion preceding Lemma 2.5 , and by that lemma each $P * Q * R_{i}$ is proper.

In any extension by $P * Q, E$ is a family of $m$ pairwise disjoint sets. We claim that there is an $i^{*}<m$ such that $S_{j}-f^{-1 \prime \prime} E(i)$ is stationary for every $j<n$. Otherwise, as $n<m$, there would be $i_{0}<i_{1}<m$ and $j<n$ such that both $S_{j}-f^{-1 \prime} E\left(i_{0}\right)$ and $S_{j}-f^{-1 \prime} E\left(i_{1}\right)$ were nonstationary, and so the union of these two sets would be nonstationary, but would also contain $S_{j}$, a contradiction.

We claim further that forcing with $R_{i^{*}}$ preserves the stationarity of each $S_{j}$. For in the extension by $P * Q, S_{j}-f^{-1 \prime \prime} E\left(i^{*}\right)$ is stationary. So if $r \Vdash$ "B is club in $\omega_{1}$," then we can find a countable $N \prec H\left(\omega_{2}\right)$ (taken in the extension by $P * Q$ ) containing $r, R_{i^{*}}$, and $\mathrm{B}$, such that $\alpha \in S_{j}$ but $f(\alpha) \notin E\left(i^{*}\right)$, where $\alpha=N \cap \omega_{1}$. Choose $\left\langle r_{k}\right.$ : $k<\omega\rangle$ a descending sequence in $R_{i^{*}} \cap N$ such that $r_{0}=r$, and for every dense subset $D$ of $R_{i^{*}}$ in $N$ there is a $k$ with $r_{k} \in D$. Let $r^{*}=\bigcup\left\{r_{k}: k<\omega\right\} \cup\{(\alpha, \alpha)\}$. Then $r^{*} \leq r$ and $r^{*} \Vdash$ " $\alpha \in \mathbf{B}$." So the set of conditions forcing $S_{j}$ to remain stationary is dense in $R_{i^{*}}$.

In $V$ we can find $\left(p_{0}, q_{0}\right)$ forcing each $S_{j}-f^{-1 "} E\left(i^{*}\right)$ to be nonstationary. Replacing $P * Q$ with $\left\{(p, q):(p, q) \leq\left(p_{0}, q_{0}\right)\right\}$, we may assume $\left(p_{0}, q_{0}\right)$ is trivial. Thus each term $\mathbf{S}_{j}$ is forced to denote a stationary set by every condition in $P * Q * R_{i^{*}}$. 
Let $A$ be the set of all conditions $(p, q, r) \in P * Q * R_{i}$. so that for some $\alpha<\omega_{1}$ and some $\zeta<\omega_{2}, \operatorname{lh}(p) \leq \zeta+\omega+1$ and $(p, q) \Vdash$ "f $(\alpha)=\zeta$." Given any condition $(p, q, r)$ we can find $\lambda$ and $D$ as in Lemma 2.5(b), and $N \in D$ such that $(p, q, r) \in N$. Letting $\alpha=N \cap \omega_{1}$ and $\zeta=\sup \left(N \cap \omega_{2}\right)$, we see by Lemma $2.5(\mathrm{~b})$ that $(p, q, r)$ has an extension in $A$. So $A$ is dense in $P * Q * R_{i^{*}}$. Therefore $A$ (with the ordering inherited from $P * Q * R_{i^{*}}$ ) is a proper partial order.

Let $\mathbf{C}$ be the canonical term for the club adjoined by $R_{i^{*}}$. Pick $\lambda$ regular and so large that $P * Q * R_{i^{*}},\left\langle\mathbf{D}_{\sigma}: \sigma<\omega_{1}\right\rangle,\left\langle\mathbf{S}_{i}: i<\right.$ $n\rangle$, f, and $\mathrm{C}$ all belong to $H(\lambda)$. Let $M \prec H(\lambda)$ contain all these sets, contain all countable ordinals, and have cardinality $\aleph_{1}$. Using $\mathrm{PFA}^{+}(n)$, find an $M$-generic filter $H_{0}$ on $A$ such that for each $j<$ $n, \mathbf{S}_{j}\left(H_{0}\right)$ is stationary. Let $H$ be the upward closure of $H_{0}$ in $P * Q * R_{i^{*}}$. Then $H$ is an $M$-generic filter on $P * Q * R_{i^{*}}$, each $\mathbf{S}_{j}(H)$ is stationary, and every element of $H$ has an extension in $H \cap A$.

For $i<m$ let $p^{*}(i)=\bigcup\{p(i): \exists q, r(p, q, r) \in H\}$. Let $\beta^{*}=$ $\sup \{\operatorname{lh}(p): \exists q, r(p, q, r) \in H\}$. Clearly $p^{*}(i) \subseteq S_{0}^{2} \cap \beta^{*}$ and for each $\beta \neq \beta^{*}$ there is an $i<m$ so that $p^{*}(i) \cap \beta$ is nonstationary in $\beta$. We shall show that $p^{*}\left(i^{*}\right) \cap \beta^{*}$ is nonstationary in $\beta^{*}$, from which it follows that $p^{*}$ is in fact a condition in $P$. To this end, let $f^{*}$ be the set of all pairs $(\alpha, \zeta)$ so that there is a condition $(p, q, r) \in H$ such that $(p, q) \Vdash$ " $\mathrm{f}(\alpha)=\zeta$," and let $C^{*}$ be the set of all $\alpha<\omega_{1}$ so that there is a condition $(p, q, r) \in H$ such that $(p, q, r) \Vdash$ " $\alpha \in \mathbf{C}$." Since $H$ is pairwise compatible $f^{*}$ is an increasing function, and since $H$ is $M$-generic $f^{*}$ is continuous with domain $\omega_{1}$ and $C^{*}$ is club in $\omega_{1}$. Let $\zeta^{*}=\sup f^{*-1 "} \omega_{1}$. As $\Vdash " \mathbf{f}^{\prime \prime} \mathbf{C} \cap \mathbf{E}\left(i^{*}\right)=\varnothing, " f^{* \prime \prime} C^{*}$ is club in $\zeta^{*}$ and disjoint from $p^{*}\left(i^{*}\right)$..

We claim that $\zeta^{*}=\beta^{*}$. Assume the contrary. If $\zeta^{*}>\beta^{*}$ choose $\alpha \in C^{*}$ large enough that for every $(p, q, r) \in H, \operatorname{lh}(p)<f^{*}(\alpha)$. By the definition of $f^{*}$ and $C^{*}$ (and pairwise compatibility of $H$ ) there are $(p, q, r) \in H$ and $\zeta<\omega_{2}$ so that $f^{*}(\alpha)=\zeta$ and $(p, q, r) \Vdash$ " $\alpha \in \mathbf{C}$ and $\mathbf{f}(\alpha)=\zeta$." Now $\Vdash^{\prime} \mathbf{f}^{\prime \prime} \mathbf{C} \cap \mathbf{E}\left(i^{*}\right)=\varnothing$," so $(p, q, r) \Vdash$ " $\zeta \notin$ $\mathbf{E}\left(i^{*}\right)$." Thus $\zeta \leq \operatorname{lh}(p)$. But $\operatorname{lh}(p)<f^{*}(\alpha)=\zeta$, a contradiction. On the other hand, if $\zeta^{*}<\beta^{*}$ choose $(p, q, r) \in H, i<m$, and $\eta \in p(i)$ with $\zeta^{*} \leq \eta$. Extending if need be, we may assume that $(p, q, r) \in A$. So there are $\alpha<\omega_{1}$ and $\zeta<\omega_{2}$ such that $\operatorname{lh}(p) \leq \zeta+\omega+1$ and $(p, q) \Vdash$ " $\mathrm{f}(\alpha)=\zeta$." Clearly $f^{*}(\alpha)=\zeta$, and as $f^{*}$ is increasing, $\zeta+\omega+1 \leq f^{*}(\alpha+\omega+1)<\zeta^{*}$. Hence $\zeta^{*} \leq \eta \leq \ln (p)<\zeta^{*}$, 
another contradiction. Thus the claim is proved. Therefore $p^{*}$ is a condition in $P$. Choose a $V$-generic filter on $P$ containing $p^{*}$, and let $E$ be the mutually nonreflecting family added by this filter. As $E$ and the generic filter are equiconstructible, we may speak of the interpretation $\tau^{E}$ of an arbitrary $P$-term $\tau$ by $E$. For each $\sigma<\omega_{1}$, let $D_{\sigma}=\mathbf{D}_{\sigma}^{E}$, and for $j<n$ let $\mathbf{S}_{j}^{\prime}=\mathbf{S}_{j}^{E}$. By assumption $\left(Q^{E},\left\langle D_{\sigma}: \sigma<\omega_{1}\right\rangle,\left\langle\mathbf{S}_{j}^{\prime}: j<n\right\rangle\right)$ is an obnoxious triple in $V[E]$.

Let $G=\left\{q^{E}: \exists p, r(p, q, r) \in H\right\}$. It is not hard to show $G$ is a filter on $Q^{E}$. For each $\sigma<\omega_{1},\left\{(p, q, r): p \Vdash " q \in \mathbf{D}_{\sigma}\right.$ " $\}$ is dense in $P * Q * R_{i}$ and belongs to $M$. So, as $H$ is $M$-generic $G$ is $\left\langle D_{\sigma}: \sigma<\omega_{1}\right\rangle$-generic. Now suppose $\alpha \in \mathbf{S}_{j}(H)$. Then for some $(p, q, r) \in H,(p, q) \Vdash$ “ $\alpha \in \mathbf{S}_{j}$." Since $p \geq p^{*}, p$ belongs to the $V$-generic filter we have chosen on $P$, and so $V[E] \vDash q^{E} \Vdash$ " $\alpha \in \mathbf{S}_{j}^{\prime}$." Hence $\alpha \in \mathbf{S}_{j}^{\prime}(G)$. Thus $\mathbf{S}_{j}(H) \subseteq \mathbf{S}_{j}^{\prime}(G)$, and so $\mathbf{S}_{j}^{\prime}(G)$ is stationary.

In $V[E]$ we have found $G$, a $\left\langle D_{\sigma}: \sigma<\omega_{1}\right\rangle$-generic filter on $Q^{E}$, with $\mathbf{S}_{j}^{\prime}(G)$ stationary for each $j<n$. But this is a contradiction since $\left(Q^{E},\left\langle D_{\sigma}: \sigma<\omega_{1}\right\rangle,\left\langle\mathbf{S}_{j}^{\prime}: j<n\right\rangle\right)$ is obnoxious. The theorem is therefore proved.

REMARK. A similar argument can be given with $\omega_{2}$ replaced by any regular cardinal greater than $\omega_{1}$. Hence if $n<m, V \vDash \operatorname{PFA}^{+}(n)$, and $\kappa>\omega_{1}$ is regular, then there is a countably closed, $\kappa$-Baire partial order $P$ such that $V^{P} \vDash \operatorname{PFA}^{+}(n)$ and in $V^{P}$ there is a mutually nonreflecting family of $m$ pairwise disjoint stationary subsets of $\{\alpha<$ $\kappa: \operatorname{cf} \alpha=\omega\}$.

3. Excluding mutually nonreflection stationary sets. In [Ba] it is shown that $\operatorname{PFA}^{+}(1)$ implies that for every $\kappa \geq \omega_{2}$ with $\mathrm{cf} \kappa>\omega_{1}$, every stationary subset of $\{\alpha<\kappa: \operatorname{cf} \alpha=\omega\}$ reflects. Thus Theorem 2.6 has the immediate corollary that PFA (if consistent with ZFC) does not imply $\operatorname{PFA}^{+}(1)$. It is simple to generalize this argument to show, for any $n<m$, that $\operatorname{PFA}^{+}(n)$, if consistent, does not imply $\mathrm{PFA}^{+}(m)$. We include the proof for the sake of completeness. Let $\Gamma$ be the class of all countably closed partial orders.

THeOREM 3.1. $\operatorname{MA}^{+}(\Gamma, m)$ implies, for every $\kappa \geq \omega_{2}$ with $\mathrm{cf} \kappa>$ $\omega_{1}$, that every family of $m$ stationary subsets of $\{\alpha<\kappa: \operatorname{cf} \alpha=\omega\}$ is mutually reflecting.

Proof. Suppose $\left\langle E_{i}: i<m\right\rangle$ is a family of stationary subsets of $\{\alpha<\kappa: \operatorname{cf} \alpha=\omega\}$. Let $P$ be the Levy order for collapsing $\kappa$ 
to cardinality $\aleph_{1}$ with countable conditions, and let $f$ be a $P$-term for a continuous, increasing function from $\omega_{1}$ into $\kappa$ with range cofinal in $\kappa$. As $P$ is countably closed, $\Vdash_{P} " \forall \alpha<\omega_{1} \mathbf{f} \mid \alpha \in V$." So $D_{\alpha}=\{p \in P: \exists g p \Vdash$ "f $\mid \alpha=g$ " $\}$ is dense for each $\alpha$. For each $i<m$ let $\mathbf{A}_{i}$ be a $P$-term such that $\Vdash_{p}$ " $\mathbf{A}_{i}=\left\{\alpha<\omega_{1}: \mathbf{f}(\alpha) \in E_{i}\right\}$." Since $P$ is proper, $E_{i}$ is stationary in the extension by $P$ (by Lemma 2.4), and so $\mathbf{A}_{i}$ is forced to denote a stationary set. Applying $\operatorname{MA}^{+}(\Gamma, m)$, there is a $\left\langle D_{\alpha}: \alpha<\omega_{1}\right\rangle$-generic filter $G$ such that each $\mathbf{A}_{i}(G)$ is stationary.

Letting $f^{*}=\{(\alpha, \zeta): \exists p \in G p \Vdash$ “f $(\alpha)=\zeta "\}, f^{*}$ is an increasing, continuous function from $\omega_{1}$ into $\kappa$. Put $\eta=\sup \operatorname{ran}\left(f^{*}\right)$. Note that $\operatorname{ran}\left(f^{*}\right)$ is club in $\eta$. Thus for any club $C$ in $\eta, f^{*-1 \prime \prime} C$ is club in $\omega_{1}$. Choosing $\alpha \in f^{*-1 "} C \cap \mathbf{A}_{i}(G)$, we have $f^{*}(\alpha) \in C \cap E_{i}$. So each $E_{i} \cap \eta$ is stationary, and $\left\langle E_{i}: i<m\right\rangle$ reflects mutually at $\eta$.

MM can be substituted for $\operatorname{MA}^{+}(\Gamma, 1)$ in the hypothesis, as is shown by Theorem 9 of [FMS]: MM implies, for every regular $\kappa \geq$ $\omega_{2}$, that every stationary subset of $\{\alpha<\kappa: \operatorname{cf} \alpha=\omega\}$ contains a closed set of order type $\omega_{1}$ (and so reflects). (It is not hard to prove the conclusion for every $\kappa \geq \omega_{2}$ with cf $\kappa>\omega_{1}$, given this theorem for regular $\kappa$.)

Corollary 3.2. (a) For any $n<m<\aleph_{1}, \operatorname{PFA}^{+}(n)$ does not imply $\mathrm{PFA}^{+}(m)$ (unless of course $\mathrm{PFA}^{+}(n)$ is inconsistent).

(b) PFA does not imply MM (unless PFA is inconsistent).

Proof. (a) By Theorem 2.6, if $\mathrm{PFA}^{+}(n)$ is consistent then there is a model of $\mathrm{PFA}^{+}(n)$ in which there is a family of $m$ mutually nonreflecting stationary subsets of $\omega_{2}$. Were $\operatorname{PFA}^{+}(n)$ to imply $\operatorname{PFA}^{+}(m)$, in this model there could be, by Theorem 3.1, no such family.

(b) Again, by theorem 2.6 (take $n=0$ and $m=1$ ), if PFA is consistent then it has a model in which there is a nonreflecting stationary subset of $\omega_{2}$. But if PFA implied MM then, by Theorem 9 of [FMS], there could be no such model.

\section{REFERENCES}

[Ba] J. Baumgartner, Applications of the proper forcing axiom, in the Handbook of Set-theoretic Topology (K. Kunen and J. Vaughan, editors), North-Holland, Amsterdam, (1984), 913-959.

[Be] R. Beaudoin, On Uncountable Trees and Linear Orders, doctoral dissertation, Dartmouth College (unpublished), 1984. 
[FMS] M. Foreman, M. Magidor, and S. Shelah, Martin's maximum, saturated ideals, and non-regular ultrafilters, part 1, Ann. of Math., 127 (1988), 1-47.

[Sh] S. Shelah, The semi-proper forcing axiom implies Martin's maximum but not PFA $^{+}$, J. Symbolic Logic, 52 (1987), 360-367.

[To] S. Todorčević, Trees and linearly ordered sets, in the Handbook of Set-theoretic Topology (K. Kunen and J. Vaughan, editors), North-Holland, Amsterdam, (1984), 235-293.

[V] B. Veličković, Forcing axioms and stationary sets, to appear.

Received August 29, 1989.

AUBURN UNIVERSITY

AUBURN UNIVERSITY, AL 36849-5310 


\section{PACIFIC JOURNAL OF MATHEMATICS EDITORS}

\author{
V. S. VARADARAJAN \\ (Managing Editor) \\ University of California \\ Los Angeles, CA 90024-1555-05 \\ Herbert Clemens \\ University of Utah \\ Salt Lake City, UT 84112 \\ THOMAS ENRIGHT \\ University of California, San Diego \\ La Jolla, CA 92093
}

R. FINN

Stanford University

Stanford, CA 94305

HeRmann FlaschKa

University of Arizona

Tucson, AZ 85721

VAUGHAN F. R. JoNES

University of California

Berkeley, CA 94720

STEVEN KERCKHOFF

Stanford University

Stanford, CA 94305
C. C. MOORE

University of California

Berkeley, CA 94720

MaRTIN SCHARLEMANN

University of California

Santa Barbara, CA 93106

HAROLD STARK

University of California, San Diego

La Jolla, CA 92093

\section{ASSOCIATE EDITORS}
R. ARENS
E. F. BECKENBACH
B. H. NeumanN
F. WolF
K. YosHIDA

\section{SUPPORTING INSTITUTIONS}

UNIVERSITY OF ARIZONA

UNIVERSITY OF BRITISH COLUMBIA

CALIFORNIA INSTITUTE OF TECHNOLOGY

UNIVERSITY OF CALIFORNIA

MONTANA STATE UNIVERSITY

UNIVERSITY OF NEVADA, RENO

NEW MEXICO STATE UNIVERSITY

OREGON STATE UNIVERSITY
UNIVERSITY OF OREGON

UNIVERSITY OF SOUTHERN CALIFORNIA

STANFORD UNIVERSITY

UNIVERSITY OF HAWAII

UNIVERSITY OF TOKYO

UNIVERSITY OF UTAH

WASHINGTON STATE UNIVERSITY

UNIVERSITY OF WASHINGTON 


\section{Pacific Journal of Mathematics}

Vol. 149, No. $1 \quad$ May, 1991

Takao Akahori and Harunori Ameku, On the Romanov kernel and Kuranishi's $L^{2}$-estimate for $\bar{\partial}_{\mathrm{b}}$ over a ball in the strongly pseudo convex

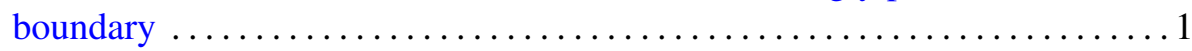

Robert Emile Beaudoin, The proper forcing axiom and stationary set

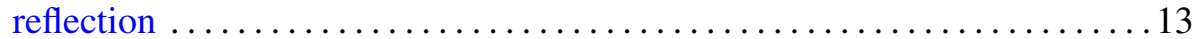

Hans Engler, A matrix Volterra integrodifferential equation occurring in

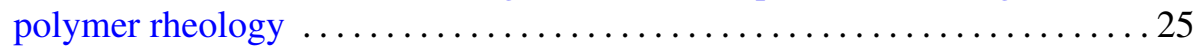

Zhong Ge, On a constrained variational problem and the spaces of

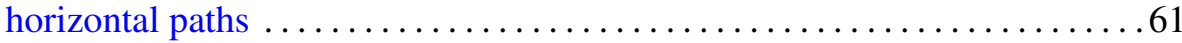

Yutaka Hemmi, Higher homotopy commutativity of $H$-spaces and the

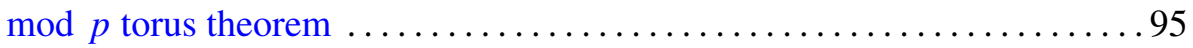

G. D. Johnson, An intrinsic characterization of a class of minimal surfaces

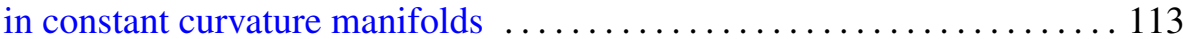

Min Ho Lee, Conjugates of equivariant holomorphic maps of symmetric domains

Jacek Nikiel, H. Murat Tuncali and Edward D. Tymchatyn, On the rim-structure of continuous images of ordered compacta

Tara Lynn Smith, Generalized Clifford-Littlewood-Eckmann groups . . . . . 157

Tara Lynn Smith, Generalized Clifford-Littlewood-Eckmann groups II:

Linear representations and applications 185 